\title{
The moderating influence of variability on belief revision
}

\author{
BOB REHDER and REID HASTIE \\ University of Colorado, Boulder, Colorado
}

\begin{abstract}
The variability of the information on which a belief was initially based should moderate the degree to which the belief is revised when new disconfirming information is received. Reasoning from everyday intuitions, psychological theories of concept representation, and a rational mathematical analysis, we inferred that as variability increased, resistance to disconfirming information would also increase. Participants' judgments of the central tendencies of distributions of numerical information confirmed this prediction.
\end{abstract}

The traditional view of stereotypes and many other conceptual generalizations is that they are rigidly held and not closely connected to the real world to which they refer (e.g., Allport, 1954; Lippmann, 1922; cf. Malt, 1995). An alternate view is that stereotypes are useful cognitive structures which people are usually justified in maintaining. McCauley, Stitt, and Segal (1980) have argued that many social stereotypes are adaptive and reliable, and that the absence of stereotype revision in the face of disconfirming evidence may be rational (see also Anderson, 1991).

Determining when a person is clinging irrationally to a category abstraction is a complicated business. One factor that should influence the strength of the link between evidence and the stereotypic generalization and, hence, the probability of belief revision is the variability of the values of attributes that are the original basis of the generalization (Thagard \& Nisbett, 1982). We hypothesize that the more variable a group is, the less should be and will be the impact of disconfirming information on the general impression of the group. For example, if a person's primary source of knowledge about a group is memories of individual group members, then the exemplars of the more variable group will be more dispersed than those of the less variable group. When a new disconfirming group member is encountered, it will be similar to more members of the highly variable group than it will be to the less variable group, and thus will appear "less disconfirming" for the more variable group. Alternatively, if variability information is abstracted into a range of typical values (or most extreme values), then new disconfirming group members are more likely to fall inside the range of the more variable group, again leading to the result that beliefs about more variable groups are less likely

Preparation of this article was supported by National Science Foundation Grant SES 9308369. Correspondence should be directed to B. Rehder, Department of Psychology, Campus Box 345, University of Colorado, Boulder, CO 80309 (e-mail: rehder@psych.colorado. edu). to be disconfirmed than beliefs about less variable groups. (Intuitive arguments such as these are supported by formal analysis, but space limits preclude the publication of our analyses in the present article. Contact the authors for information concerning the formal analysis.)

Many empirical studies have shown that people remember variability information and use that information in a variety of judgments (e.g., Fried \& Holyoak, 1984; Nisbett \& Kunda, 1985; Park \& Hastie, 1987; Peterson \& Beach, 1967). Variability has been shown to influence the propensity with which individuals are willing to generalize an attribute from a group member to the group as a whole (Nisbett, Krantz, Jepson, \& Kunda, 1983; Park \& Hastie, 1987; Quattrone \& Jones, 1980) and to apply an attribute from the group to one of its members (Lambert, 1995; Ryan, Judd, \& Park, 1996). But, we know of only one study of the effect of variability on belief revision. Hewstone, Johnston, and Aird (1992) found more change in trait ratings following presentation of stereotypeinconsistent information about the occupational category "teachers" than the category "accountants." They interpreted this as evidence that stereotype change was likelier to occur for heterogeneous than for homogeneous categories, although they were cautious about the generality of this conclusion.

The purpose of the present study was to determine whether variability affects the degree to which judgments of the central tendency of a group or concept are revised when disconfirming information is received. Subjects were first provided with information about a category that they were told was collected in 1985 . They were then provided with descriptions of a few category members collected in 1995 (the "disconfirmers"), which differed substantially from the central tendency of the category in 1985 , and were asked to judge what the mean of the category was likely to be in 1995 . We manipulated the variability of the 1985 information, and hypothesized, on intuitive and rational grounds, that the magnitude of revision in estimates of the mean would decrease as the variability of the group increased. 


\section{METHOD}

\section{Subjects}

Thirty-two University of Colorado undergraduates from an introductory psychology course received course credit for participating in this experiment.

\section{Design}

A $2 \times 4 \times 4 \times 2$ factorial design was employed. The first factor, varied between subjects, was the variability (low or high) of the original (1985) distribution of attribute values presented. The second factor, varied within subjects, was the domain from which the distributions were drawn, henceforth referred to as cases A, B, C, and D. The final two factors were between-subjects counterbalancing factors; case presentation order was varied and, for each case, the new (1995) disconfirming instances appeared at either the upper or the lower end of the scale.

\section{Materials}

A case consisted of an attribute of a group, an original (1985) distribution of values of that attribute (low- and high-variability versions), and a new (1995) distribution (with lower and upper end versions). The group, attribute, units in which the attribute was presented, and distributions for cases A, B, C, and D are presented in Table 1. For each case, the standard deviation of the high-variability version of the 1985 distribution is three times that of the low-variability version. Within each case, the absolute distance of the mean of the new 1995 distribution from the mean of the original distribution is the same in the low- and high-variability conditions. In units of standard deviations of the original distribution, however, the distance between orig. inal and new means is 3 in the low-variability condition and 1 in the high-variability condition. The 1995 distributions shown in Table 1 are in their upper end versions, where the new mean is greater than the original mean (e.g., for case A, the upper end version mean was 378 , the lower end version mean was 312 ).

Cases A, B, C, and D represent cases of "change," where the new distribution differs from the original one. In order to balance these cases with cases of "no change," subjects were also presented with four no-change filler cases; the first example case (of nine received) was always a change case.

The subjects were presented with samples of instances drawn from the distributions shown in Table 1 and those for the four filler cases and the example case (32 instances from the original 1985 distributions and 3 instances from the new 1995 distributions). Instances were drawn at random, subject to the constraint that the resulting sample was perfectly representative of the underlying population: The mean and standard deviation of the sample were equal to those statistics for the population.

\section{Procedure}

Subjects produced judgments for the nine cases presented by computer in the following order: The example case, filler case 1, filler case 2 , first target case, filler case 3 , second target case, filler case 4 , third and fourth target cases. The task was to determine whether groups had changed since 1985, and, if so, by how much. A case began with a description of the group and the attribute (e.g., "Blood Potassium Levels of the People of Thailand"), followed by 32 examples from 1985 in the form of numbers followed by the unit of measurement (e.g., "7.3 milligrams"). The examples were presented on the screen one at a time, each appearing $2.0 \mathrm{sec}$ with a blank screen intervening for $0.3 \mathrm{sec}$. The computer then prompted for the mean of the 1985 distribution and the lower and upper limits of a $95 \%$ confidence interval

The rest of the information entered for a case concerned the new 1995 distribution. First, in order to determine subjects' beliefs about the likelihood and direction of change over a 10 -year period, the subject was asked to estimate the 1995 distribution (the mean and lower and upper limits of the $95 \%$ confidence interval) given no disconfirming information. The computer also prompted for a confidence judgment from 1 to 9 , indicating whether or not the distribution had changed

Next, given exposure to one 1995 individual, the subjects were asked to estimate the 1995 distribution. This individual was presented on the screen in the same manner as the 1985 examples had been. The subjects were told, "We are interested in whether you think the 1995 individual is different enough to warrant the conclusion that the group has changed, and if so by how much." The subjects then entered the mean, the $95 \%$ confidence interval, and a confidence judgment. Then the subjects were presented with a second and finally a third $1995 \mathrm{ex}-$ ample, and were asked to estimate the distribution and produce a confidence judgment after each.

After completing the example case and before continuing on with the remaining eight cases, the subjects were told that the cases were randomly selected by computer, and that, "All eight cases might indicate change, or all eight might indicate no change, or some might indicate change and others not."

\section{RESULTS}

To make cases comparable, subjects' estimates of the means and the lower and upper limits of the $95 \%$ confidence intervals were converted into $z$ scores of the highvariability version of the original distributions. As a result of this transformation, the mean of the original distribution for every case is assigned a value of 0 . To analyze subjects' judgments concerning $95 \%$ confidence intervals, the size of the interval was computed by subtracting the $z$ score of the upper limit from the $z$ score of the lower limit. (The lower and upper limits of the $95 \%$ confidence intervals estimated by the subjects were approximately symmetrical around their estimates of means.) There was no significant effect of presentation order of cases in any analysis, and the results have been collapsed over that factor.

Table 1

Group, Attribute, Unit of Measurement, and Original 1985 and New 1995 Distributions for Cases A, B, C, and D

\begin{tabular}{|c|c|c|c|c|c|c|c|c|}
\hline \multirow[b]{3}{*}{ Case } & \multirow[b]{3}{*}{ Group } & \multirow[b]{3}{*}{ Attribute } & \multirow[b]{3}{*}{ Unit } & \multicolumn{5}{|c|}{ Distribution } \\
\hline & & & & \multicolumn{3}{|c|}{ Original } & \multicolumn{2}{|c|}{ New } \\
\hline & & & & $M$ & Low $S D$ & High $S D$ & $M$ & $S D$ \\
\hline A & Fraternity & $\begin{array}{c}\text { Amount of oxydilic } \\
\text { mineral in diet }\end{array}$ & Milligrams & 345 & 11 & 33 & 378 & 10.7 \\
\hline $\mathrm{B}$ & Shreeble nests & Diameter & Centimeters & 48 & 3 & 9 & 57 & 3 \\
\hline $\mathrm{C}$ & New Zealanders & Light sensitivity & Candelas & 165 & 8 & 24 & 189 & 8 \\
\hline $\mathrm{D}$ & Fiction novels & Length & $\begin{array}{r}\text { Thousands } \\
\text { of words }\end{array}$ & 64 & 6 & 18 & 82 & 6 \\
\hline
\end{tabular}

Note--The new 1995 distributions are shown in the upper end form. 


\section{Original Distribution Estimates}

In general, estimates of the mean were fairly accurate: For low-variability subjects, the estimates were -0.05 , $-0.06,-0.03$, and -0.03 for cases $\mathrm{A}, \mathrm{B}, \mathrm{C}$, and $\mathrm{D}$, respectively; the corresponding estimates for the highvariability subjects were $-0.04,-0.17,-0.05$, and -0.07 . These estimates were subjected to one-sample $t$ tests to determine whether or not they differed from the true mean of 0 . In only the two conditions involving case B did the test approach or reach significance $[t(15)=$ $-2.28, p<.05$, and $t(15)=-2.05, p<.10$, for the lowand high-variability conditions, respectively].

Estimates of the size of the $95 \%$ confidence interval were sensitive to our manipulation of the true variability of the original distributions: For low-variability subjects, the size estimates were $1.11,1.05,1.06$, and 1.01 , for cases A, B, C, and D; for high-variability subjects, they were $3.46,3.48,3.08$, and 3.13. A $2 \times 4$ analysis of variance (ANOVA) on variability and case yielded a large effect of variability $[F(1,30)=72.96, p<.0001]$. Estimates of the interval sizes were relatively inaccurate, systematically underestimating the true sizes, and the underestimation was greater in the high-variability condition $(.66$ of the true size) than in the low-variability condition (.81 of the true size).

\section{Original Distribution Versus Initial Estimate of the New Distribution}

Subjects were asked to estimate the new 1995 distribution given no information other than the passage of 10 years. These estimates did not differ significantly from the estimates produced for the original 1985 distribution. Thus, subjects were agnostic about whether the distributions had changed over the 10 -year period.

\section{New Distribution Estimates}

The true mean of the new distribution was less than the original mean in half the cases, and greater than the original mean in the other half. To make these cases comparable, the $z$ scores for the former cases were negated (the result of this transformation is to assign a value of 1 to the new mean in every case).

The subjects' transformed estimates of the new distribution are presented in Table 2 for the low- and highvariability subjects, for each of 0 (the initial estimate based on just the passage of 10 years), 1,2 , and 3 disconfirmers. ${ }^{\prime}$ Estimates of the new mean are clearly influenced by the amount of disconfirming evidence received $(-0.02,0.20,0.38$, and 0.51 for $0,1,2$, and 3 disconfirming instances, respectively). In addition, estimates of the new mean were influenced by the variability of the original distribution $(0.34$ for low-variability vs. 0.19 for the high-variability subjects). A $2 \times 2 \times$ $4 \times 4$ ANOVA with variability condition and lower and upper end counterbalancing as between-subjects factors and case and number of disconfirmers as within-subject factors revealed statistically reliable effects of the number of disconfirmers $[F(3,28)=90.20, p<.0001]$ and variability condition $[F(1,28)=9.72, p<.005]$ and their interaction $[F(3,28)=4.36, p<.005]$. Figure 1 suggests that the effect of the number of disconfirmers on estimates of the mean is linear, and that the strength of the linear relationship is conditional on variability. This conclusion is supported by statistical analysis: The singledegree-of-freedom test of the linear trend of the number of disconfirmers was highly significant $[F(1,28)=$ $129.14, p<.0001]$ and interacted with variability condition $[F(1,28)=5.47, p<.05]$. However, there was also a small, but significant, quadratic effect of the number of disconfirmers $[F(1,28)=4.80, p<.05]$. The quadraticeffect result reflects the fact that the magnitude of the change going from zero disconfirmers to one $(0.22)$ is greater than the change going from one to two $(0.18)$, which is greater than the change going from two to three $(0.13)$. Thus, each subsequent disconfirmer carries less weight in the revision of the estimate of the new mean. This quadratic effect did not interact with variability condition $[F(1,28)=2.08, p=.16]$.

Table 2

Mean Estimates, 95\% Confidence Interval Sizes, and Confidence Judgments for the New 1995 Distributions in the Presence of 0, 1, 2, and 3 Disconfirmers

\begin{tabular}{|c|c|c|c|c|c|c|c|c|c|c|c|c|}
\hline \multirow[b]{2}{*}{ Case } & \multicolumn{3}{|c|}{$\begin{array}{c}0 \text { Disconfirmers } \\
\text { (Initial } 1995 \text { Estimate) }\end{array}$} & \multicolumn{3}{|c|}{1 Disconfirmer } & \multicolumn{3}{|c|}{2 Disconfirmers } & \multicolumn{3}{|c|}{3 Disconfirmers } \\
\hline & Mean & Size & Confidence & Mean & Size & Confidence & Mean & Size & Confidence & Mean & Size & Confidence \\
\hline \multicolumn{13}{|c|}{ Low Variability } \\
\hline$A$ & -0.06 & 1.09 & 5.19 & 0.21 & 1.18 & 5.69 & 0.43 & 1.22 & 5.81 & 0.56 & 1.21 & 6.44 \\
\hline $\mathrm{B}$ & -0.02 & 1.27 & 4.94 & 0.32 & 1.44 & 5.81 & 0.56 & 1.54 & 6.31 & 0.73 & 1.31 & 6.50 \\
\hline $\mathrm{C}$ & -0.03 & 1.16 & 5.19 & 0.31 & 1.27 & 6.19 & 0.51 & 1.07 & 6.50 & 0.71 & 1.13 & 7.13 \\
\hline $\mathrm{D}$ & 0.02 & 1.06 & 5.13 & 0.26 & 1.28 & 5.75 & 0.45 & 1.28 & 5.94 & 0.52 & 1.26 & 6.25 \\
\hline$M$ & -0.02 & 1.15 & 5.11 & 0.28 & 1.29 & 5.86 & 0.49 & 1.28 & 6.14 & 0.63 & 1.23 & 6.58 \\
\hline \multicolumn{13}{|c|}{ High Variability } \\
\hline A & -0.08 & 3.70 & 5.13 & 0.07 & 3.41 & 5.19 & 0.27 & 3.03 & 5.81 & 0.41 & 2.97 & 6.63 \\
\hline B & 0.12 & 3.83 & 4.75 & 0.18 & 3.30 & 4.75 & 0.30 & 3.35 & 4.81 & 0.41 & 3.04 & 5.31 \\
\hline $\mathrm{C}$ & -0.05 & 3.20 & 4.81 & 0.10 & 3.08 & 5.19 & 0.28 & 2.97 & 5.88 & 0.39 & 2.80 & 5.88 \\
\hline $\mathrm{D}$ & -0.09 & 3.11 & 4.94 & 0.11 & 3.08 & 4.50 & 0.30 & 3.04 & 4.81 & 0.41 & 2.89 & 4.75 \\
\hline$M$ & -0.03 & 3.46 & 4.91 & 0.12 & 3.22 & 4.91 & 0.28 & 3.10 & 5.33 & 0.39 & 2.92 & 5.64 \\
\hline
\end{tabular}




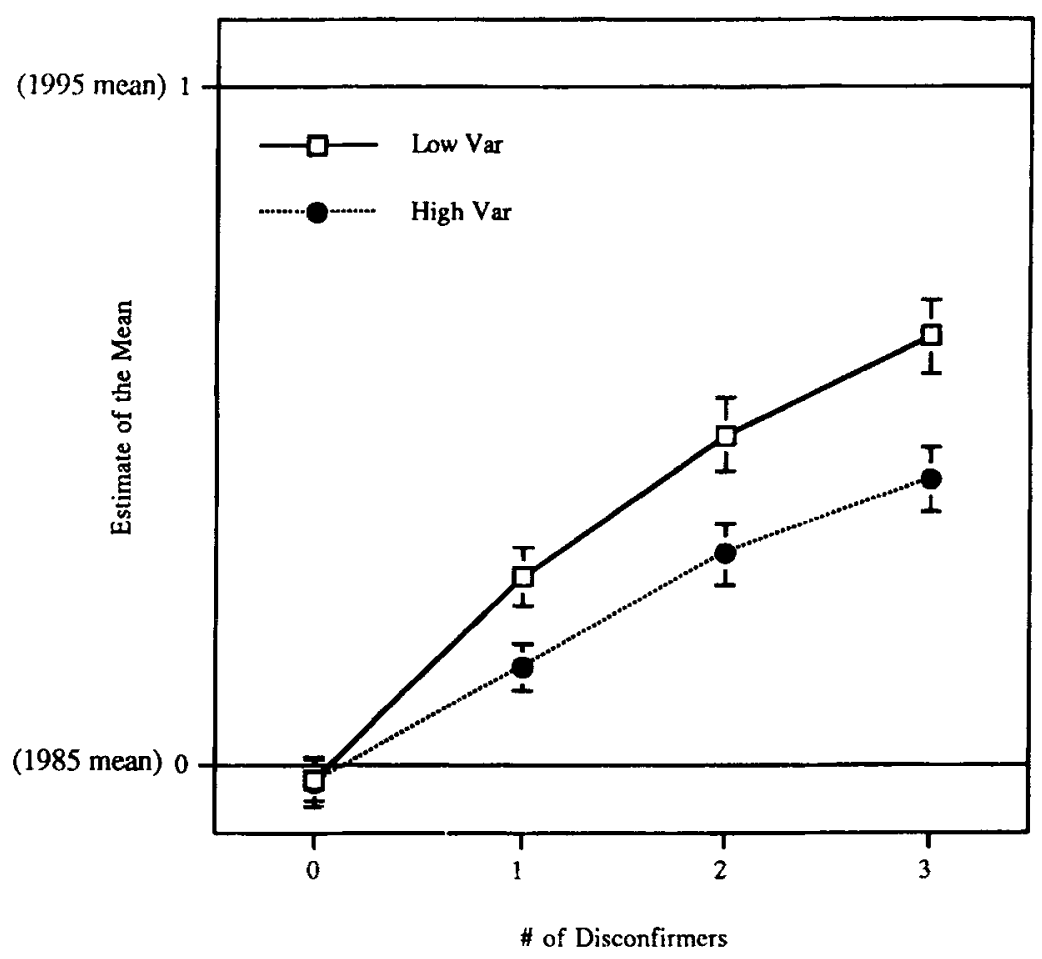

Figure 1. Mean estimates of the low- and high-variability groups as a function of the number of disconfirmers.

Since the appearance of gradual updating in Figure 1 might be a result of averaging over subjects, the changes in mean estimates as a function of disconfirmers were examined for each subject individually (averaged over the four cases). Of 32 subjects, 21 followed a strategy of gradual updating, with each of the 3 disconfirmers causing at least some change in the estimate of the mean in the direction of the true 1995 mean and only 2 subjects who could be described as following a nonlinear strategy. Thus, we conclude that the smooth pattern of change suggested by Figure 1 is not an artifact of averaging over subjects.

The effects of disconfirmers and variability on estimates of the mean were mirrored in subjects' confidence ratings: For low-variability subjects the confidence ratings were $5.11,5.86,6.14$, and 6.58 for cases $\mathrm{A}, \mathrm{B}, \mathrm{C}$, and $\mathrm{D}$, respectively; for high-variability subjects, they were 4.91 , $4.91,5.33$, and 5.64. A $2 \times 2 \times 4 \times 4$ ANOVA revealed main effects of the number of disconfirmers $[F(3,28)=$ $14.35, p<.0001]$ and the variability condition $[F(1,28)=$ $4.51, p<.05]$; their interaction was marginally significant $[F(3,28)=2.02, p<.15]$. Just as for estimates of the mean, the effect of the number of disconfirmers on confidence judgments is largely linear, as supported by the significant test of the linear trend $[F(1,28)=18.42$, $p<.001]$.

\section{DISCUSSION}

The results of this study empirically confirm our prediction: The more variable a group is, the less likely it is that the impression of the group's central tendency will be revised. We obtained this result consistently across four content domains with 1,2 , and 3 disconf irmers. Our results are different from those reported by Hewstone et al. (1992), but there are so many differences between the two methods (e.g., we used numerical stimuli, while they used written descriptions of behaviors, and we manipulated the distribution of instances initially presented to our subjects, while they manipulated the general category label [teachers vs. accountants]) that further research will be required to determine the exact sources of the discrepancies.

Our subjects were fairly accurate in judging means of distributions of presented numbers, but relatively inaccurate in judging $95 \%$ confidence intervals. On average, our subjects underestimated the sizes of these confidence intervals. In our data, the standard errors associated with the estimates of the lower and upper limits of $95 \%$ confidence intervals were twice as large as those for estimates of the mean, suggesting that our subjects were unpracticed at judging variability.

The results from the present study are consistent with an image of belief revision as a gradually adjusting "bookkeeping" process (Rothbart, 1981; Weber \& Crocker, 1983). The updating strategies of only 2 of 32 subjects were consistent with an alternate discontinuous, allat-once revision process. Most of our subjects exhibited patterns of adjustment and sensitivity to variability that would fit a "qualitative Bayesian" model (Camerer, 1994; but see Lopes, 1985).

Our demonstration of the moderating effect of variability on belief change has a number of intriguing implications for the revision of social stereotypes: (1) Beliefs about an in-group will be more resistant to change in the presence of disconfirming evidence than are those about an out-group. (2) Beliefs about a group learned through personal experience with member exemplars will be more resistant to change than those learned through an abstract group description. And (3) beliefs about a group with meaningful subtypes will be more resistant to change than beliefs about groups without them. This research suggests an interesting dilemma: Complex mental representations about groups that include laudable beliefs about the variability of a group may be the reason that inaccuracies about the group are maintained in the presence of all but overwhelming disconfirmations. 


\section{REFERENCES}

ALLPORT, G. W. (1954). The nature of prejudice. Garden City, NJ: Doubleday.

ANDERSON, J. R. (1991). The adaptive nature of human categorization. Psychological Review, 98, 409-429.

CAMERER, C. (1994). Individual decision making. In J. H. Kagel \& A. E. Roth (Eds.), The handbook of experimental economics (pp. 587703). Princeton, NJ: Princeton University Press.

FrIED, L. S., \& HolYOAK, K. J. (1984). Induction of category distributions: A framework for classification learning. Journal of Experimental Psychology: Learning, Memory, \& Cognition, 10, 234-257.

Hewstone, M., Johnston, L., \& AiRD, P. (1992). Cognitive models of stereotype change: (2) Perceptions of homogeneous and heterogeneous groups. Journal of Experimental Social Psychology, 22, 235 . 249.

LAMBERT, A. J. (1995). Stereotypes and social judgment: The consequences of group variability. Journal of Personality \& Social Psychology, 68, 388-403.

LippmanN, W. (1922). Public opinion. New York: Harcourt, Brace.

LoPEs, L. L. (1985). Averaging rules and adjustment processes in Bayesian inference. Bulletin of the Psychonomic Society, 23, 509-512.

MALT, B.C. (1995). Category coherence in cross-cultural perspective. Cognitive Psychology, 29, 85-148.

McCauley, C., Stitt, C. L., \& Segal, M. (1980). Stereotyping: From prejudice to prediction. Psychological Bulletin, 87, 195-208.

NisbetT, R. E., Krantz, D. H., Jepson, C. J., \& Kunda, Z. (1983). The use of statistical heuristics in everyday inductive reasoning. Psychological Review, 90, 339-363.

NisbetT, R. E., \& Kunda, Z. (1985). Perception of social distributions. Journal of Personality \& Social Psychology, 48, 297-311.
Park, B., \& Hastie, R. (1987). The perception of variability in category development: Instance- versus abstraction-based stereotypes. Journal of Personality \& Social Psychology, 53, 621-635.

Peterson, C. R., \& BEACH, L. R. (1967). Man as an intuitive statistician. Psychological Bulletin, 68, 29-46.

Quattrone, G. A., \& JoNEs, E. E. (1980). The perception of variability within in-groups and out-groups: Implications for the law of small numbers. Journal of Personality \& Social Psychology, 38, 141-152.

RothbaRT, M. (1981). Memory processes and social beliefs. In D. L. Hamilton (Ed.), Cognitive processes in stereotyping and intergroup behavior (pp. 145-182). Hillsdale, NJ: Erlbaum.

RYAN, C. S., JUdD, C. M., \& PARK, B. (1996). Effects of racial stereotypes on judgments of individuals: The moderating role of perceived group variability. Journal of Experimental Social Psychology, 32, 71-103.

THAGARD, P., \& NisbetT, R. E. (1982). Variability and confirmation. Philosophical Studies, 42, 379-394.

WEBER, R., \& CROCKER, J. (1983). Cognitive processes in the revision of stereotypic beliefs. Journal of Personality \& Social Psychology, $45,961-977$

\section{NOTE}

1. Some effects of the upper end, lower end counterbalancing factor reached significance, but did not support general interpretations. They are therefore omitted from this report.

(Manuscript received January 8,1996 ; revision accepted for publication May 6, 1996.) 